A THEORETICAL STUDY OF THE ELECTRON-PROTON INSTABILITY IN A LONG PROTON PULSE*

AUTHOR(S):

Tai-Sen F. Wang

AOT-1

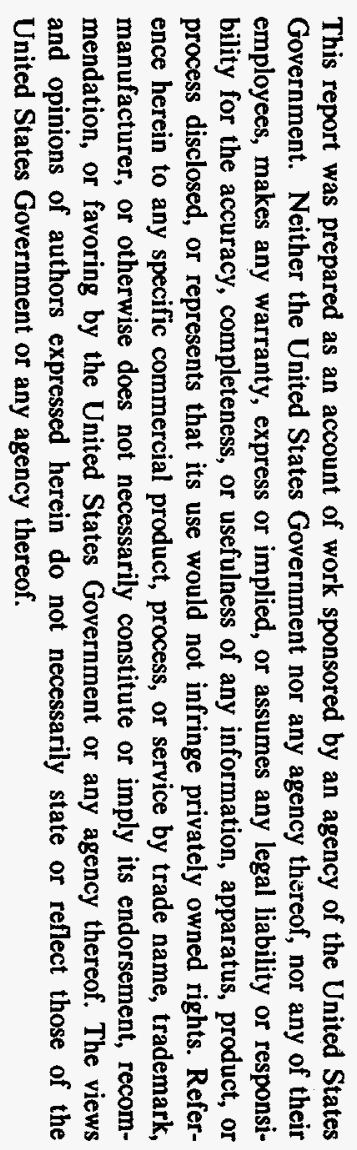

SUBMITTED TO:

1995 Particle Accelerator Conference

Dallas, Texas, May 1-5, 1995
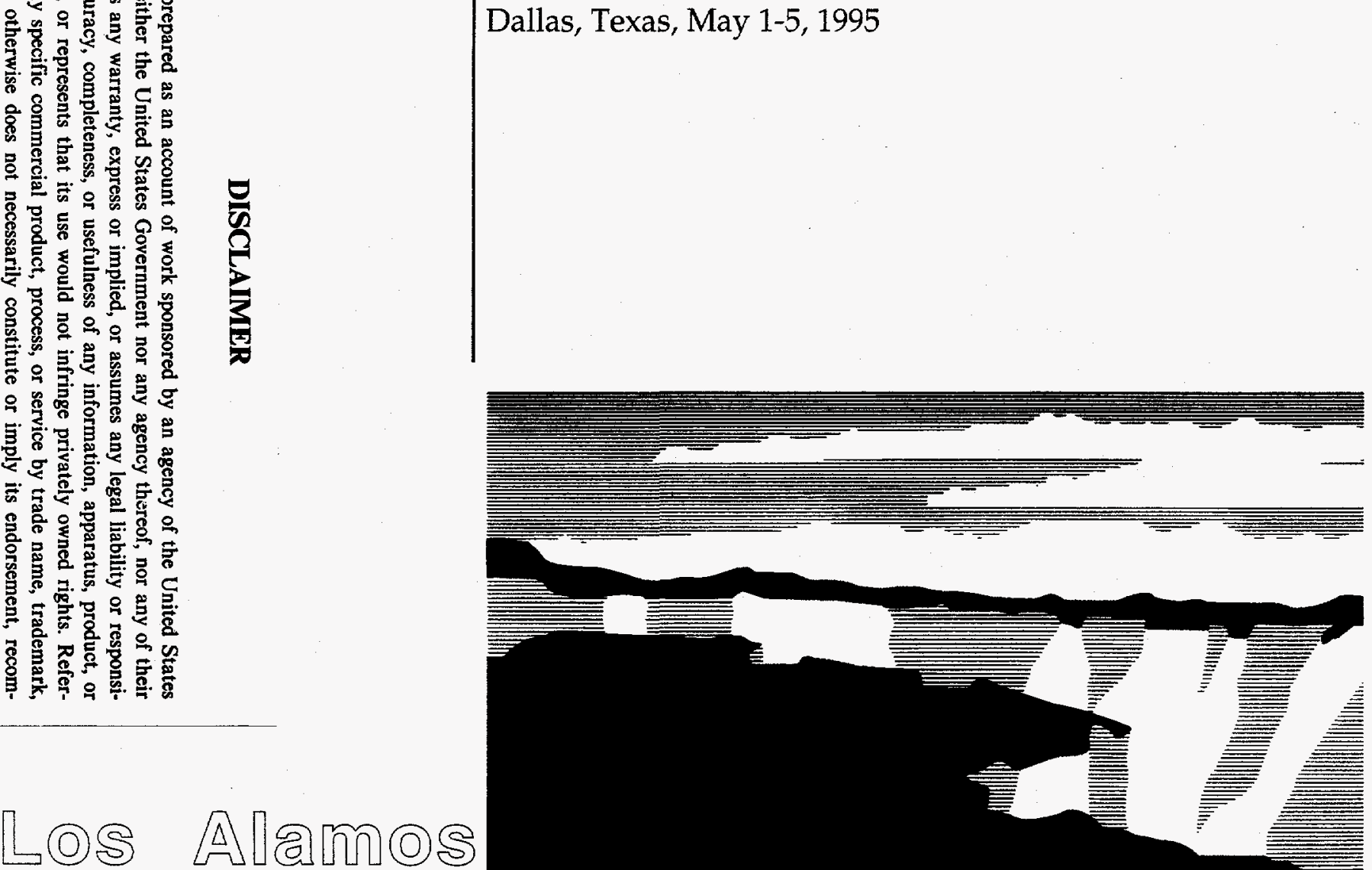

NATIONAL LABORATORY

Los Alamos National Laboratory, an affirmative action/equal opportunity employer, is operated by the University of California for the U.S. Department of Energy under contract W-7405-ENG-36. By acceptance of this anticle, the publisher recognizes that the U.S. Government retains a nonexclusive, royalty-free license to publish or reproduce the published form of this contribution, or to allow others to do so, for U.S. Government purposes. The Los Alamos National Laboratory requests that the publisher identify this article as work performed under the auspices of the U.S. Department of Energy. 


\section{DISCLAIMER}

Portions of this document may be illegible in electronic image products. Images are produced from the best available original document. 


\title{
A THEORETICAL STUDY OF THE ELECTRON-PROTON INSTABILITY IN A LONG PROTON PULSE'
}

\author{
Tai-Sen F. Wang \\ AOT-1, MS H808, Los Alamos National Laboratory \\ Los Alamos, New Mexico 87545, USA
}

\section{Abstract}

The electron-proton instability of a long, intense, and partially neutralized proton bunch is studied by numerically solving the equations of motion for the line centroid of the proton beam and the line centroid of the trapped electrons. The formalism takes into account the effects of variable line densities and alternatinggradient focusing. Good qualitative agreement between the computational results and experimental observations was obtained when applying the theory to the Los Alamos Proton Storage Ring (PSR). Both the case of a clean extraction gap and the case with a few percent of protons in the extraction gap were studied. It is found that with only a few percent neutralization, the PSR beam can become unstable in both cases. The same equations and method were used to study the stability of the proton beam in the accumulator ring of the proposed LANSCE II spallation-neutron source. The results indicate that the $e$ $p$ instability can also occur in the LANSCE II accumulator ring for only a few percent neutralization.

\section{INTRODUCTION}

Coherent transverse instability has been observed in the PSR in both bunched and unbunched beams.[1-3] Spectrum analyses of the vertical beam position monitor (BPM) signals from unstable beams indicate that the peak of the frequency distribution may vary from several ten $\mathrm{MHz}$ to about three hundred $\mathrm{MHz}$. The fast growth of the instability and the dependence of the frequency spectrum peak on the beam conditions suggest that the instability is more likely caused by the trapped electrons in the proton bunch instead of fixed-frequency impedances. The results from a previous injection-foil biasing experiment and a beam-shaking experiment on stabilizing the marginally stable beams also suggest that the instability in the PSR could be aroused by the electrons trapped in the proton beam.

Since the $e-p$ instability has been previous observed in the Bevatron at LBL[4,5] and at CERN [6-8] and now appears to occur in the PSR, precaution must be taken in designing the next generation spallation neutron sources such as the Los Alamos LANSCE II and the European Spallation Source (ESS) to avoid such kind of instability.

This report documents a theoretical investigation on

*Work supported by Los Alamos National Laboratory Institutional Supporting Research, under the auspices of the US Department of Energy. the $e-p$ instability in a long proton bunch like the one in the PSR or in the proposed LANSCE II. We will present the results of solving the coupled equations of motion for the centroid of the proton beam and the centroid of the trapped electrons. An example will be given for PSR.

\section{THEORETICAL MODEL}

We consider a bunched proton beam of total length $L$ with a round cross-section of radius $a$, propagating with a constant speed $v$ inside a perfect conducting pipe of radius $b$. Protons are focused in the transverse direction by an external force that depends linearly on the radial distance. A Cartesian coordinate system is adopted such that the $z$-axis is in the direction of proton propagation and the $y$-axis is perpendicular to the ring. The origin of the coordinate system coincides with the center of the beam cross section. The proton bunch is partially neutralized by electrons possibly produced by secondary emission, gas ionization, or the charge-change injection process. We assume that in the equilibrium state, electrons are trapped in the proton beam (no electrons between beam and pipe), and both species of particles are distributed uniformly in the transverse direction. Both the population line-densities of protons and electrons, $\lambda_{p}$ and $\lambda_{e}$, can be functions of $z$ along the proton beam. We also assume that the proton beam is unstable against the perturbation in only one transverse direction, so we need to concentrate only in one direction of the transverse motion, say the $y$-direction. The axial motion of electrons and the synchrotron motion of protons are neglected for simplicity. The study of the $e-p$ instability here is based on the investigation of the motion of the line centroid of the proton beam $Y_{p}(z, t)$ and the line centroid of the trapped electrons $Y_{e}(z, t)$ defined as the averaged displacements of the electrons and and protons, respectively, at the location $z$ and at the time $t$.

Taking the perturbation into account, we can derive the following two equations for $Y_{p}$ and $Y_{e}$ by averaging the equations of motion for single particles and adding the damping terms(e.g. from energy spread, tune spread, and non-linear forces):

and

$$
\begin{gathered}
\left(\frac{\partial}{\partial t}+v \frac{\partial}{\partial z}\right)^{2} Y_{p}-C_{d p}\left(\frac{\partial Y_{P}}{\partial t}+v \frac{\partial Y_{P}}{\partial z}\right) \\
+\omega_{\beta}^{2} Y_{p}=\left(\frac{2 g \lambda_{p} r_{p} \chi c^{2}}{a^{2} \gamma}\right) Y_{e}, \\
\frac{d^{2} Y_{e}}{d t^{2}}-C_{d e} \frac{d Y_{e}}{d t}+\omega_{e}^{2} Y_{e}=\left(\frac{2 g \lambda_{p} r_{e} c^{2}}{a^{2}}\right) Y_{p},
\end{gathered}
$$

where 


$$
\omega_{\beta}^{2}=\omega_{\beta o}^{2}+\frac{2 \lambda_{p} r_{p} c^{2}}{a^{2}}\left[\frac{\chi}{\gamma}-\frac{1}{\gamma^{3}}\left(\frac{a}{b}\right)^{2}\right]
$$

$C_{d p}$ and $C_{d e}$ are the damping constants for the proton and electron motions, respectively, $\omega_{\beta o}$ is the betatron frequency due to the external focusing force only, $\omega_{e}^{2}=$ $\omega_{e o}^{2}(1-\chi), \omega_{e o}=(c / a) \sqrt{2 \lambda_{p} r_{e}}$ is the bouncing frequency of electrons, $r_{p}$ and $r_{e}$ are the classical radii of a proton and an electron, respectively, $\gamma=\left(1-v^{2} / c^{2}\right)^{-1 / 2}, c$ is the speed of light, $\chi(z)=\lambda_{e}(z) / \lambda_{p}(z)$ is the fraction of neutralization, and $g=1-(a / b)^{2}$, is the geometric factor of the beam environment. We note that $\omega_{e o}$ is a function of $z$, and all nonlinear forces have been neglected in deriving Eqs. (1) and (2).

Eq. (1) is formulated for a continuous transverse focusing case. A similar equation can be derived for an AG focusing channel by replacing $\omega_{\beta o}^{2}$ in Eq. (1) by $\pm(q v G) /\left(\gamma m_{p}\right)$ within quadrupoles and 0 for drift spaces, where $q$ is the unit charge, $G$ is the quadrupole field gradient, and $m_{p}$ is the rest mass of a proton. Except for a few very special cases, it is difficult to solve Eqs. (1) and (2) analytically in general; a numerical approach has to be used.

\section{NUMERICAL RESULTS AND EXAMPLE}

Equations (1) and (2) were solved numerically with given system parameters, damping constants, line densities, and assumed initial perturbations for the stability study. The evolution of the system was examined at various time intervals. An example is given below. The emphasize here will be on the qualitative results rather than the quantitative results.

As an example, we consider a case in PSR for no particles in the gap between bunches. All electrons are created within the the proton bunch and subsequently lost in the beam gap of the same turn. We adopted a parabolic distribution for the proton line density:

$$
\lambda_{p}(z)=6 N_{p} s(1-s) / L,
$$

where $s=z / L$, and $z$ is the axial distance measured from the head of the bunch. We assumed that the number of electrons produced by the proton beam is accumulated over the bunch length and the number of electrons trapped locally is proportional to the product of the all created electrons and the proton line density. If we further assume that the electron generation per proton is constant around the ring and the electron production rate is proportional to $\lambda_{p}(z)$, we can infer that

$$
\lambda_{e}(z)=0.016 s^{2}(3-2 s) \lambda_{p}(z),
$$

where the neutralization is arbitrarily chosen to be $1.22 \%$ at $z \approx 0.68 L$. The density profiles $\lambda_{p}$ and $\lambda_{e}$ are shown in Fig. 1 as functions of $\tau=v z$.

We used the following PSR parameter values for computation: $\gamma=1.85, a=0.015 \mathrm{~m}, b=0.05 \mathrm{~m}$, the circumference $C=90 \mathrm{~m}, N_{p}=2.74 \times 10^{13}$, and
$L=65 \mathrm{~m}$. The maximal electron bouncing frequency, at these parameter values is about $189 \mathrm{MHz}$. A set of lattice parameters simplified from that of the PSR was implemented in the computation. The quadrupole strength was adjusted to yield a betatron tune of 2.3 close to that in the PSR. The values of $C_{d p}$ and $C_{d e}$ were chosen as $1.4 \times 10^{5} / \mathrm{s}$ (estimated from kinetic theory) and $1.9 \times 10^{8} / \mathrm{s}$ (one e-folding in one bouncing period), respectively. We assumed that initially $Y_{e}(z, 0)=0$, and $Y_{p}(z, 0)=0.076 \sin [(2 \pi z) /(2.1 \mathrm{~m})]$. The unit of $Y_{p}$ and $Y_{p}$ is arbitrary length. This chosen sinusoidal $Y_{p}$, when carried by the traveling proton beam, corresponds to a wave oscillating about $120 \mathrm{MHz}$ in time.

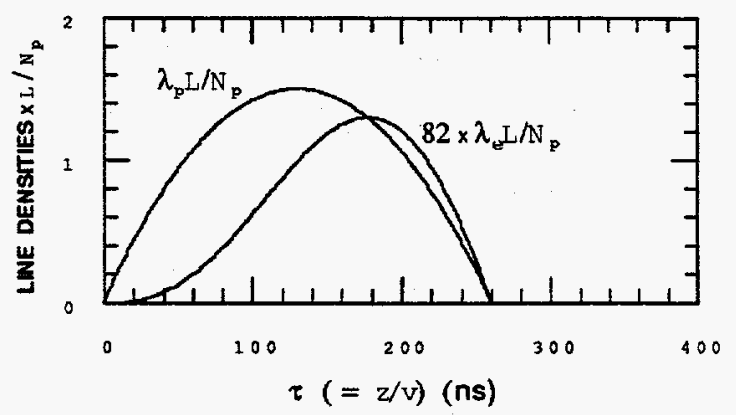

Fig. 1. The line densities used in the example shown here after normalized by $N_{p} / L$. The electron line density has been multiplied by a factor of 82 . The gap is between $\tau=260 \mathrm{~ns}$ and $\tau=360 \mathrm{~ns}$.

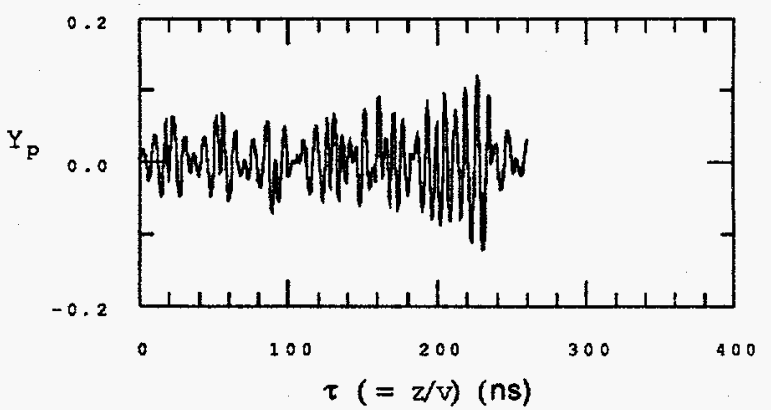

Fig. 2. The snap-shots of $Y_{p}$ after tracking the motions for a time interval equivalent to that of 37 revolutions of protons in PSR $(\approx 13.3 \mu \mathrm{s})$. The vertical axis has arbitrary unit.

In Fig. 2, we show the snap-shots of $Y_{p}$ after tracking the motions for a time interval equivalent to that of 37 revolutions of protons in PSR $(\approx 13.3 \mu \mathrm{s})$. At this stage, the wiggles of the electron bouncing frequencies have been developed in the proton bunch. One sees the amplitude of the oscillation in the tail of the bunch has been increased. This shows that the instability grows both in time and distance.

We also computed the simulated scope signal

$$
S(\tau)=\left.\frac{\partial\left[\lambda_{p}(v \tau) Y_{p}(v \tau, t)\right]}{\partial t}\right|_{t=\tau}
$$

for comparing with the experimental observations, where the derivative with respect to $t$ is to take into account the differential effect of the BPM in the PSR. The simulated scope signal corresponding to the displacement shown in Fig. 2 is given in Fig. 3 to compare with a real BPM 
signal of an unstable beam in the PSR shown in Fig. 4. In both figures, we see higher frequency wiggles in the middle of the bunch where the proton density is high, and lower frequency wiggles at the ends of the bunch where the proton density is low.

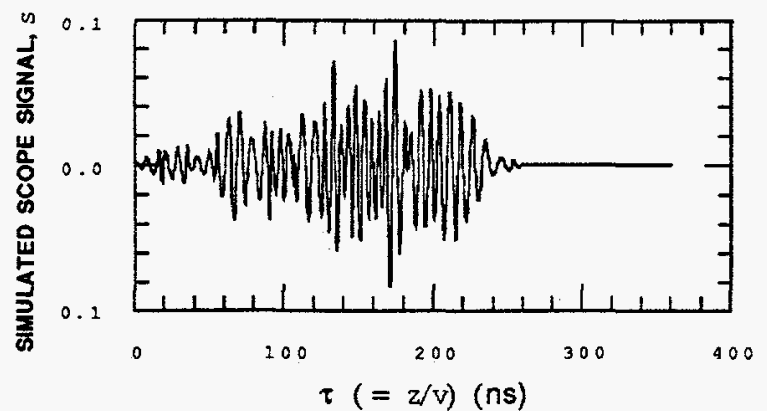

Fig. 3. The simulated the scope signals corresponding to the displacement shown in Fig. 2.

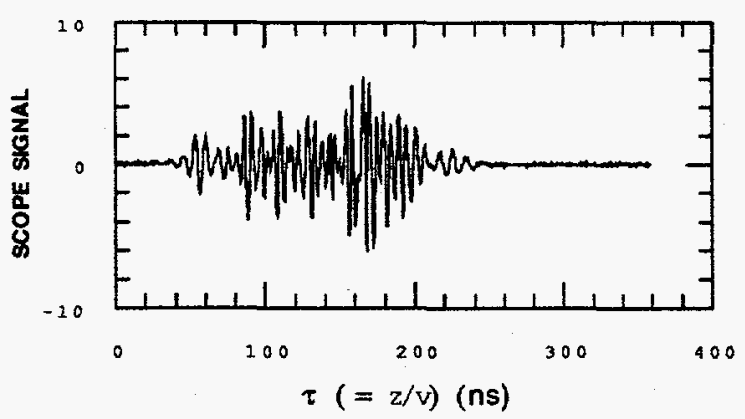

Fig. 4. A BPM signal of an unstable beam in the PSR.

The general properties observed in the numerical solutions are summarized in the following:

(i) In the parameter ranges of PSR and LANSCE II, for reasonably chosen damping rates of electrons and protons, it takes only a few percent of neutralization in the proton beam for the $e-p$ instability to develop. The growth time can range from a few to a few tens of micro-seconds.

(ii) A gap empty of proton does not always ensure the beam stability, and the multi-turn trapping of electrons is not a necessary condition for the e-p instability to develop. If a proton beam bunch is sufficiently neutralized (a few percent in the PSR and LANSCE II cases), the $e-p$ instability still may occur even when the gap is empty. The instability grows slightly faster and the stability threshold is slightly lower when there are a small number of protons in the gap.

(iii) The wavelength of the $e-p$ oscillation varies with the proton line density. Short wavelength in the high density region and long wavelength in the low density region. In a proton bunch of non-uniform density, the frequency spectrum is relatively wide. This is a distinct characteristic of the oscillations that involve more than one species of particles.

(iv) The $e-p$ instability grows both in time and space.
Therefore, most of the oscillation growth occurs in the later part or in the tail of a proton bunch.

(v) We have studied the situation of non-uniform electron production around the ring. Comparing with the case of uniform electron production, we found that for the same number of electrons, the $e-p$ oscillation is slightly more unstable when the electrons production is concentrated in part of the ring.

(vi) We found that a proton bunch is more unstable and has a higher growth rate in an AG focusing system than in a continuous, uniform focusing system.

(vii) In several cases, we have included a low-level random noise at the beginning of each turn to check its effects. We observed that the noise can slightly lower the instability threshold and increase the growth rate.

\section{CONCLUSIONS}

We have studied the electron-proton instability in a long proton bunch by solving the equations of motion for the centroid of the proton beam and the centroid of the trapped electrons. For PSR, we have examined both the cases of filled gap and empty gap. We found that in either case a few percent neutralization can lead to instabilities of fast growth rates. We found that the threshold is lower and the growth rate is higher when the gap is filled in. In the numerical solutions, we saw the wavelength of the $e-p$ oscillation depends on the local proton line density. Wavelengths are long (short) in the low (high) density region. Therefore the frequency spectrum can be widebanded. Our numerical results seem to agree well with the experimental observations qualitatively. We have also found that both the AG focusing and random noise can lower the threshold and increase the growth rate of the $e-p$ instability. For LANSCE II, we also found that the threshold of neutralization for the e-p instability is only a few percent.

\section{REFERENCES}

[1] T. Wang et al., Proc. of 1993 Particle Accelerator Conf., IEEE Catalog No. 93CH3279-7, Vol. 5, p. 3297.

[2] D. Neuffer et al., Nucl. Instr. and Meth. A321, 1 (1992).

[3] E. Colton et al., Proc. of 1991 IEEE Particle Accelerator Conf., IEEE Catalog No. 91CH3038-7, Vol. 3, p. 1896.

[4] H. Grunder and G. Lambertson, Proc. 8th Intl. Conf. on High Energy Accelerators, CERN, 1971, p. 308.

[5] L. J. Laslett, A. M. Sessler and D. Möhl, Nucl. Instrum. Meth., 121, 517 (1974).

[6] H. G. Hereward, CERN Internal Report 71-15 (1971).

77 E. Keil and B. Zotter, CERN Internal Note CERNISR-TH/71-58, December 1971.

[8] H. Schönauer and B. Zotter, CERN Internal Note, May 1972. 